\section{Military Technical College Kobry El-Kobbah, Cairo, Egypt.}

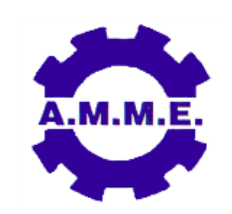

$17^{\text {th }}$ International Conference on Applied Mechanics and Mechanical Engineering.

\title{
DYNAMICS AND MEASURING OF FEET FORCE DISTRIBUTIONS OF SIX-LEGGED ROBOT
}

\author{
A. S. Zaghloul*, W. M. Hussein* and A. Badawy*
}

\begin{abstract}
This paper presents a detailed dynamic modeling of phantom ax12 six-legged robot. The direct and inverse kinematic analysis for each leg has been considered in order to develop an overall kinematic model of the robot, when it walks with tripod gait in a straight path. Newton-Euler formulation has been utilized to determine the joint torques. This study also aims to estimate feet force distributions of the said robot using minimization of norm of feet forces, which is necessary for its real-time control. These forces were compared with the real reaction forces measured from the robot.
\end{abstract}

\section{KEYWORDS}

Kinematics, Dynamics, Feet force distributions, Six-legged robot

\footnotetext{
${ }^{*}$ Egyptian Armed Forces.
} 


\section{INTRODUCTION}

Walking machines are desirable because they can navigate terrain features that are similar in size to the size of the robot, whereas wheeled and tracked vehicles are only suitable for obstacles smaller than half the diameter of the wheel. Furthermore, if given an ability to find locally horizontal footholds in regionally steep terrain, they can climb extreme angles. Applications potentially include reaching territories which are unreachable or dangerous for humans, exploration, mining, military, rescue, and industrial environments, on earth and beyond. Nevertheless, legged robots are also used for experimental studies on the behavior of living animals and for testing Artificial Intelligence (Al) techniques [1]

The available options in legged robots are enormous, configuration from 2 legged (Humanoid), 4 legged (Quadrupeds), 6 legged (Hexapod) and the 8 legged (Octopod) configuration like spiders [2].

Typical hexapod robots can be classified into rectangular and hexagonal ones. Rectangular hexapods inspired from insects have six legs distributed symmetrically along two sides; each side having three legs, this design is fast in forward direction but less flexible in turning, moving sideways or moving backwards.

Hexagonal hexapods have six legs distributed symmetrically around the body. The hexagonal hexapod has the advantage of being more flexible and in moving and achieving the same walking speeds in all directions see Fig. 1.

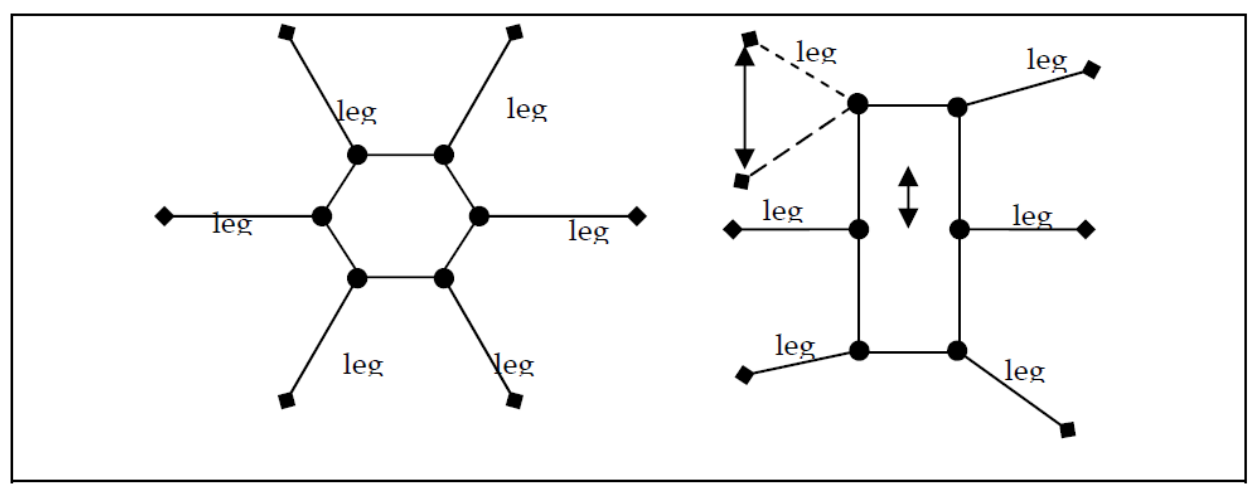

Figure 1. Two types of hexapod robots [3].

A lot of examples can be found of the six-legged robot design, we will study phantom Ax12 rectangular hexapod see Figure 2 .

In order to have a better understanding of walking and other important issues of walking, such as dynamic stability, kinematic and dynamic models based on a realistic walking robot design are necessary. Here, an attempt has been made to carry out kinematics, dynamics and feet force distributions of a realistic six-legged robot. 


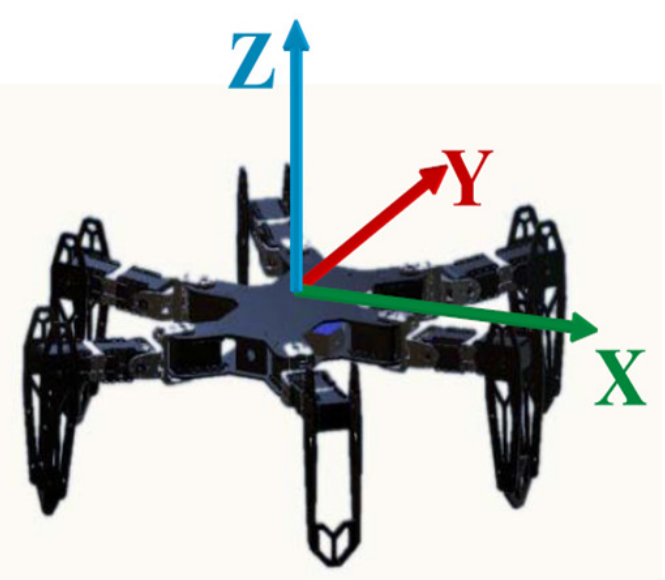

Figure 2. Phantom Ax12 rectangular hexapod.

The organization of the paper is as follows: In Section 2, kinematic model and leg trajectory generation of hexapod is presented. In Section 3, Dynamic model of Sixlegged Robot was developed. Results discussions and conclusions are in Section 4.

\section{KINEMATIC MODEL OF HEXAPOD}

The robot considered in this study (Figure 2) consists of a trunk body of rectangular cross-section and six legs, which are similar and symmetrically distributed on either sides of the trunk body. Each leg has three powered rotary joints with the typical articulated (RRR) configuration, i.e. the second and third joints' axes are parallel to each other and perpendicular to the first joint's axis. The three actuators are Dynamixel AX-12 servo motor. The Denavit-Hartenberg (D-H) notations [4] have been used in kinematic modeling of each leg (refer to Figure 3). Table 1 shows four $\mathrm{D}-\mathrm{H}$ parameters required to completely describe the leg mechanism where:

- Link length $a_{i}$ is the distance from $Z_{i}$ to $Z_{i+1}$ measured along $X_{i}$.

- Link twist $\alpha_{i}$ is the angle from $Z_{i}$ to $Z_{i+1}$ measured about $X_{i}$.

- Link offset $d_{i}$ is the distance from $X_{i-1}$ to $X_{i}$ measured along $Z_{i}$.

- Joint angle $\theta_{i}$ is the angle from $X_{i-1}$ to $X_{i}$ measured about $Z_{i}$.

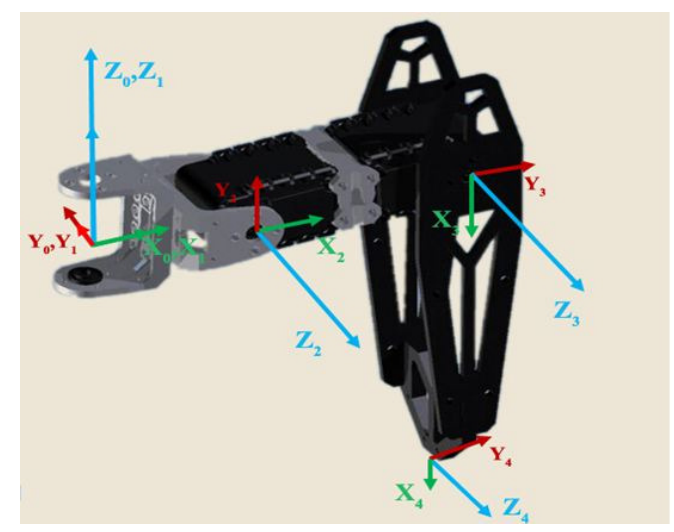

Figure 3. Robot legs with all link frames. 
Table 1. D-H parameters for three joint legs.

\begin{tabular}{c|c|c|c|c}
\hline \hline $\begin{array}{c}\text { Link } \\
\text { no/parameter }\end{array}$ & $\mathrm{a}_{\mathrm{i}-1}$ & $\mathrm{a}_{\mathrm{i}-1}$ & $\mathrm{~d}_{\mathrm{i}}$ & $\theta_{\mathrm{i}}$ \\
\hline 1 & 0 & 0 & 0 & $\theta 1$ \\
2 & 90 & 52 & 0 & $\theta 2$ \\
3 & 0 & 82 & 0 & $-\theta 3$ \\
4 & 0 & 140 & 0 & 0 \\
\hline
\end{tabular}

\section{Forward Kinematics Problem}

The homogeneous transformation matrix [4] describing the relative translation and rotation between $\mathrm{i}^{\text {th }}$ and $(\mathrm{i}-1)^{\text {th }}$ coordinate systems is represented as follows:

$$
{ }^{i-1} T_{i}=\left[\begin{array}{cccc}
\cos \theta_{i} & -\sin \theta_{i} & 0 & a_{i-1} \\
\sin \theta_{i} \cos \alpha_{i-1} & \cos \theta_{i} \cos \alpha_{i-1} & -\sin \alpha_{i-1} & -\sin \alpha_{i-1} d_{i} \\
\sin \theta_{i} \sin \alpha_{i-1} & \cos \theta_{i} \sin \alpha_{i-1} & \cos \alpha_{i-1} & \cos \alpha_{i-1} d_{i} \\
0 & 0 & 0 & 1
\end{array}\right]
$$

Thus, foot tip reference frame $\{4\}$ can be expressed in the leg reference frame $\{0\}$ as given below:

$$
\begin{aligned}
& { }^{0} T_{4}={ }^{0} T_{1}^{1} T_{2}{ }^{2} T_{3}^{3} T_{4} \\
& =\left[\begin{array}{cccc}
\mathrm{c}\left(\theta_{2}-\theta_{3}\right) \times c \theta_{1} & -s\left(\theta_{2}-\theta_{3}\right) \times c \theta_{1} & s \theta_{1} & c \theta_{1} \times\left(140 \times \mathrm{c}\left(\theta_{2}-\theta_{3}\right)+82 \times c \theta_{2}+52\right) \\
\mathrm{c}\left(\theta_{2}-\theta_{3}\right) \times s \theta_{1} & -s\left(\theta_{2}-\theta_{3}\right) \times s \theta_{1} & -c \theta_{1} & s \theta_{1} \times\left(140 \times \mathrm{c}\left(\theta_{2}-\theta_{3}\right)+82 \times c \theta_{2}+52\right) \\
s\left(\theta_{2}-\theta_{3}\right) & \mathrm{c}\left(\theta_{2}-\theta_{3}\right) & 0 & 140 \times s\left(\theta_{2}-\theta_{3}\right)+82 \times s \theta_{2} \\
0 & 0 & 0 & 1
\end{array}\right]
\end{aligned}
$$

\section{Inverse Kinematics Problem}

As seen in the previous Section a forward kinematic problem has been solved but the reverse operation is often interesting, and if it is possible for the leg end point to reach a position in space, it is also possible to determine the angles at all the joints, for the given position To be able to find the angles of all the joints on the robot, it is necessary to know the goal position of the end points, and also the pitch, yaw, roll, and position of the CG of robot body, in the global frame.

There are two main methods to solve inverse kinematics: numerical solutions and closed-form solutions. Because of their iterative nature, numerical solutions generally are much slower than the corresponding closed-form solution. Numerical approach is suitable in more complex robots. In this work geometrical approach is used to solve the inverse kinematics problem.

Before the IK can be solved for the individual legs, the leg end point coordinates, which are referenced in the global frame, needs to be transformed to the individual 
leg frames. This inverse transformation is the inverse of the body to leg transformation ${ }^{B} T_{l}$ and global to body frame transformation ${ }^{G} T_{B}$ pseudo inverse of ${ }^{B} T_{l}$ and ${ }^{G} T_{B}$ is shown in Equations (3) and (4).

$$
\begin{aligned}
& { }^{B} T_{G}=\left({ }^{G} T_{B}\right)^{-1}=\left[\begin{array}{cc}
\left({ }^{G} R_{B}\right)^{T} & -\left({ }^{G} R_{B}\right)^{T} \times{ }^{G} d_{B} \\
0 & 0
\end{array}\right] \\
& { }^{l} T_{B}=\left({ }^{B} T_{l}\right)^{-1}=\left[\begin{array}{cc}
\left({ }^{B} R_{l}\right)^{T} & -\left({ }^{B} R_{l}\right)^{T} \times{ }^{B} d_{l} \\
0 & 0
\end{array}\right]
\end{aligned}
$$

where:

${ }^{G} R_{B}$ is the rotational transformation from the body frame to the global frame.

${ }^{6} d_{B}$ is translational transformation from the body frame to the global frame.

${ }^{B} R_{l}$ is the rotational transformation from the leg frame to the body frame.

${ }^{B} d_{l}$ is translational transformation from the leg frame to the body frame.

The following equations shows how to solve inverse kinematics:

coxa angle $\theta_{1}=\tan ^{-1} \frac{Y_{4}}{X_{4}}$
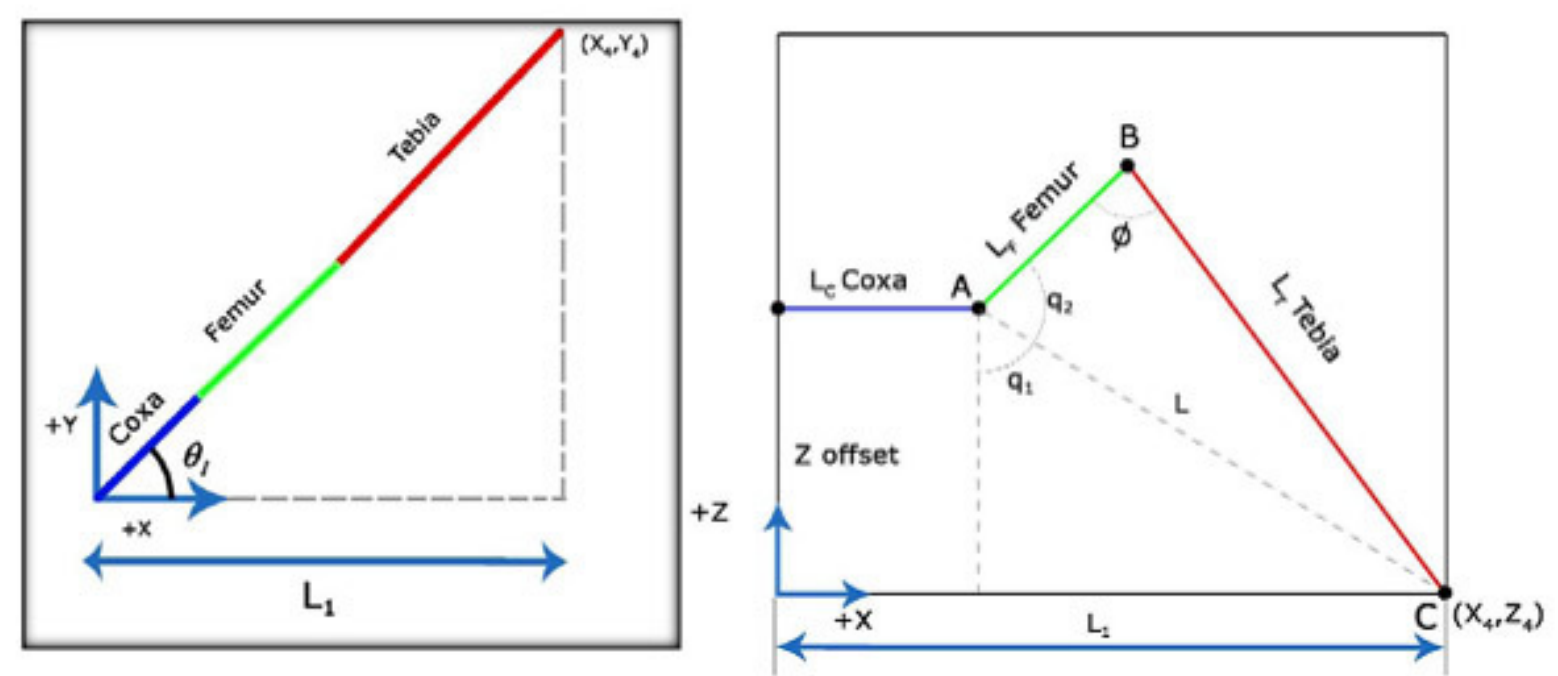

Figure 4. Illustration of inverse kinematics solution.

$$
\begin{aligned}
& \mathrm{L}_{1}=\sqrt{\mathrm{X}_{4}{ }^{2}+\mathrm{Y}_{4}{ }^{2}} \\
& \mathrm{~L}=\sqrt{\mathrm{Z}_{(\text {offset })}^{2}+\left(\mathrm{L}_{1}-\mathrm{L}_{C}\right)^{2}}
\end{aligned}
$$


where:

$\mathrm{Z}_{\text {(offset) }}$ is the body height.

$\mathrm{L}_{\mathrm{C}} \quad$ is the coxa link length.

$\mathrm{L}_{F} \quad$ is the femur link length.

$\mathrm{L}_{T} \quad$ is the tebia link length.

\section{Get femur angle above horizon}

$\mathrm{q}_{1}=\tan ^{-1} \frac{\mathrm{Z}_{(\text {offset })}}{\left(\mathrm{L}_{1}-\mathrm{L}_{C}\right)}$

$\mathrm{q}_{2}=\cos ^{-1} \frac{\sqrt{L_{F}^{2}+L^{2}-L_{T}^{2}}}{2 \times L_{F} \times L}$

$\theta_{2}($ femur angle $)=\mathrm{q}_{1}+\mathrm{q}_{2}$

Tibia angle from femur

$\varphi=\cos ^{-1} \frac{\sqrt{L_{F}^{2}+L_{T}^{2}-L^{2}}}{2 \times L_{F} \times L_{T}}$

$\theta_{3}($ tibia angle $)=\varphi-180$

\section{Leg Trajectory Planning}

In this section, we concerned with methods of computing a trajectory that describes the desired motion of robot leg in multidimensional space. Here, trajectory refers to a time history of position, velocity, and acceleration for each degree of freedom.

The robot moves straight forward at a constant velocity on flat surface with tripod gait (duty factor $\beta=1 / 2$ ) (refer to Figure 5). To ensure a smooth path to be followed, each joint trajectory followed by the swing leg joints is assumed to be governed by a fifth order polynomial which is a function of time (t) (refer to Figure 6).

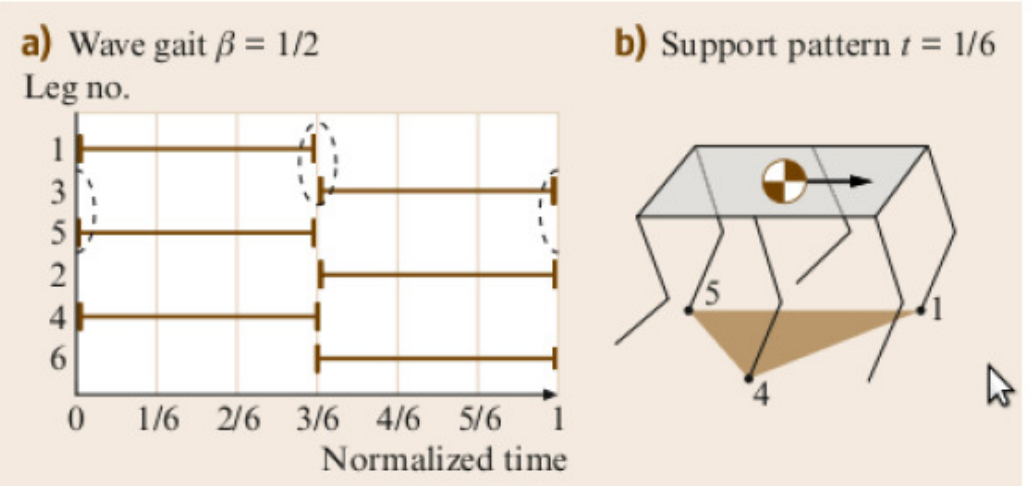

Figure 5. Tripod gait of a hexapod robot, three legs move at the same [5]. 


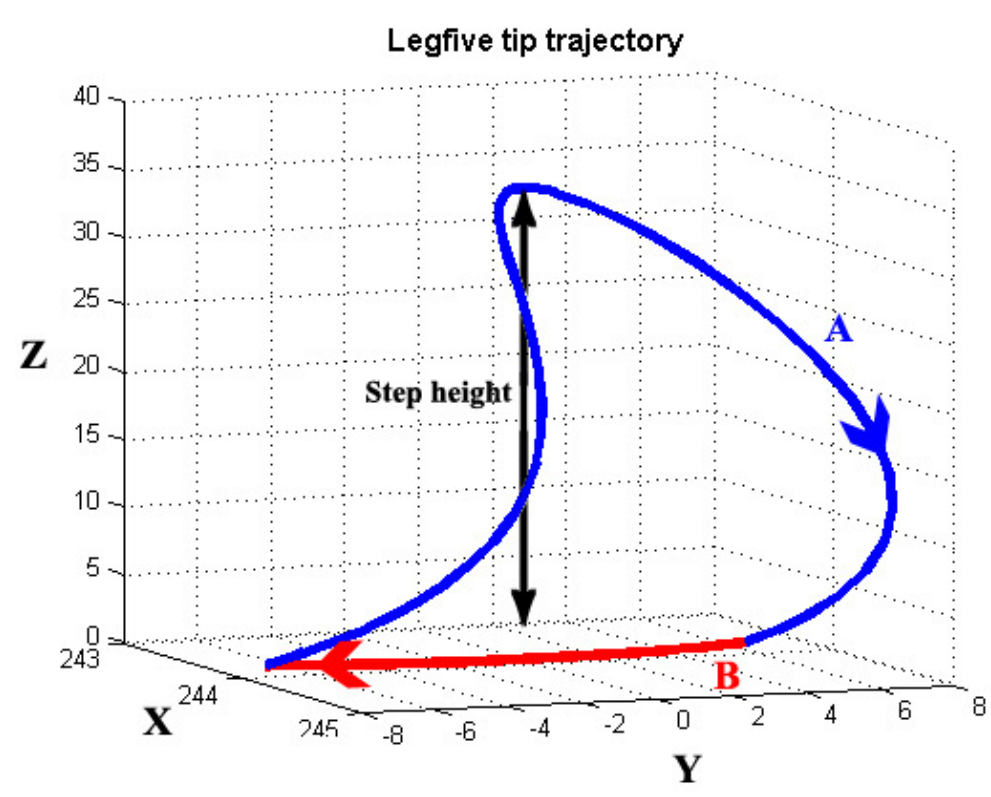

Figure 6. Leg five trajectory generation.

In order to generate trajectory in both support and transfer phases of our robot leg tip. It is required to enter the step length, step height, step time and sample, which determines the number of points in the trajectory. From first two inputs the start, via and end points are calculated. Then these three points are fed to robot IK model to find ( $\left.\theta_{1 \mathrm{~s}} \theta_{2 \mathrm{~s}} \theta_{3 \mathrm{~s}}, \theta_{1 \mathrm{~m}} \theta_{2 \mathrm{~m}} \theta_{3 \mathrm{~m}}, \theta_{1 \mathrm{e}} \theta_{2 \mathrm{e}} \theta_{3 \mathrm{e}}\right)$ where: $\theta_{\text {is }}$ is the initial value of joint $i$ of the robot leg that achieve start point of step, $\theta \mathrm{im}$ is the via piont value of joint $i$ of the robot leg that achieve step height and $\theta_{i e}$ is the end value of joint $i$ of the robot leg that achieve end point of step. Finally, the last two inputs and angles output of IK model are fed to the trajectory generation to generate a smooth trajectory between angles at the step time see Figure 7

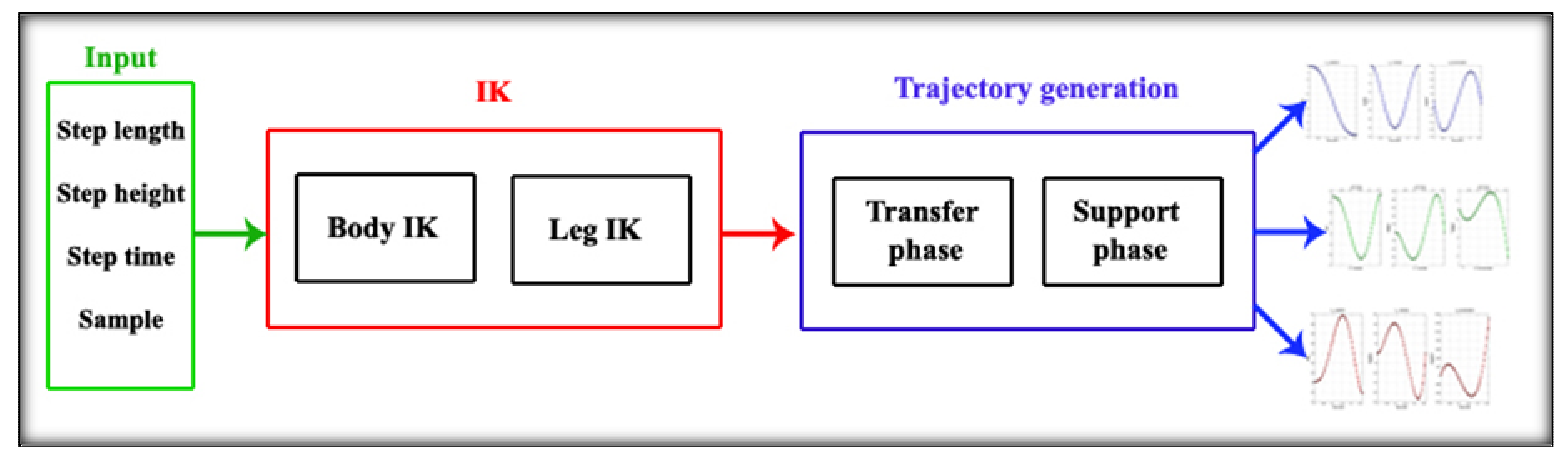

Figure 7. Illustration of leg trajectory generation process.

$$
\begin{aligned}
& \theta(t)=a_{0}+a_{1} t+a_{2} t^{2}+a_{3} t^{3}+a_{4} t^{4}+a_{5} t^{5} \\
& \dot{\theta}(t)=a_{1}+2 \times a_{2} t+3 \times a_{3} t^{2}+4 \times a_{4} t^{3}+5 \times a_{5} t^{4} \\
& \ddot{\theta}(t)=2 \times a_{2}+6 \times a_{3} t+12 \times a_{4} t^{2}+20 \times a_{5} t^{3}
\end{aligned}
$$


In order to solve equation (13) the boundary conditions of joint angles are at initial, middle and final points, joint rates and joint accelerations at initial and final points of the trajectory are applied to find the coefficients for each joint.

At the end of this part if we know the joint angles and the position and orientation of the CG of the body of the hexapod we can know the position of all leg tips we build a simulator with the help of MATLAB software to verify the kinematic model, trajectory generation and tripod gait as shown in Figure 8.

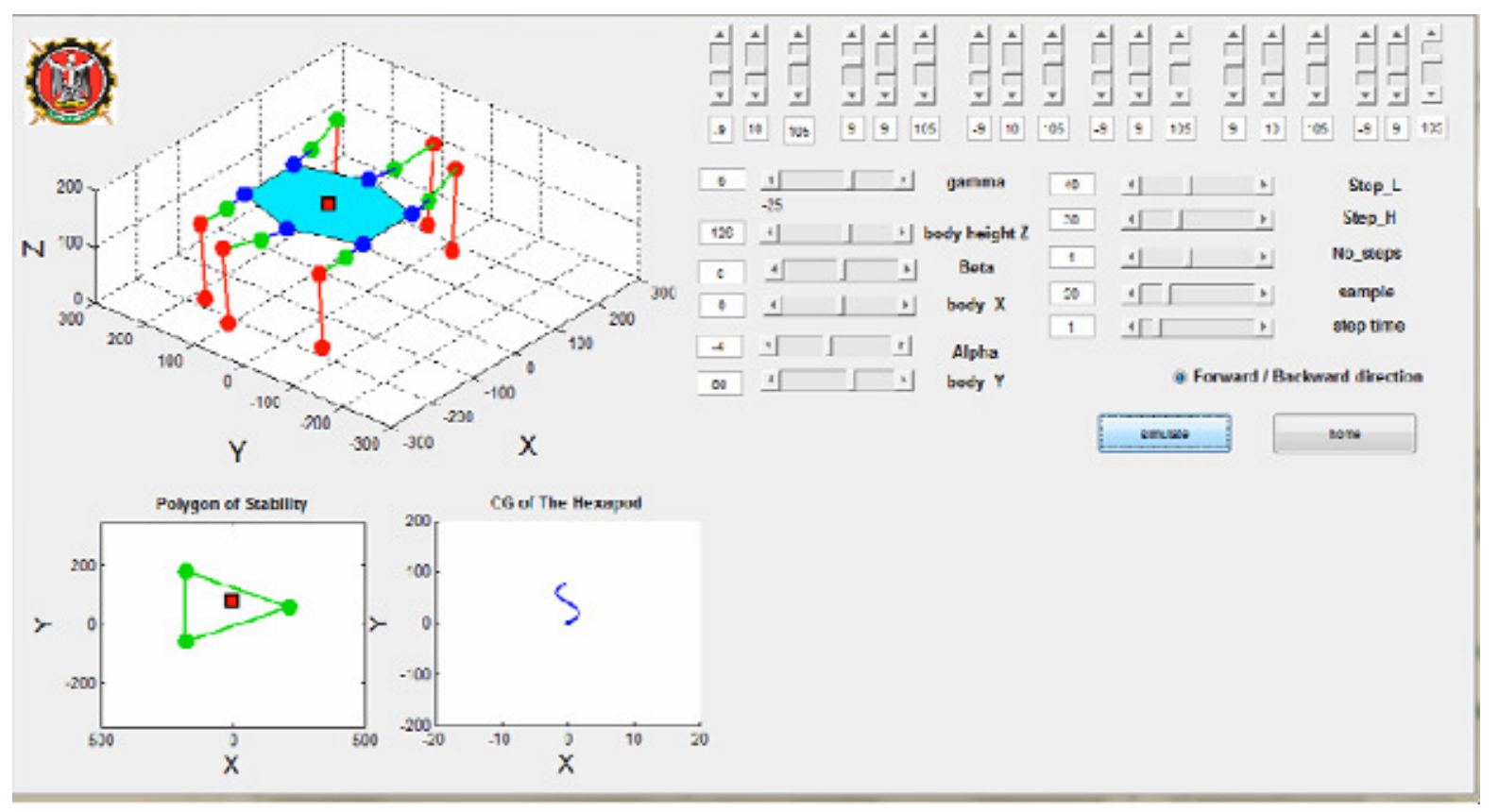

Figure 8. Hexapod robot simulator.

\section{DYNAMIC MODEL}

In order to derive the dynamic equations and finding joint torques' variations over the locomotion cycle, Newton -Euler formulation has been used. An Iterative of NewtonEuler formulation is summarized by the following equations.

Outward iterations

$$
\begin{aligned}
{ }^{i+1} \boldsymbol{\omega}_{i+1} & ={ }_{i}^{i+1} \mathbf{R}^{i} \boldsymbol{\omega}_{i}+\dot{\theta}_{i+1}{ }_{i+1} \hat{\mathbf{Z}}_{i+1} \\
{ }^{i+1} \dot{\boldsymbol{\omega}}_{i+1} & ={ }_{i}^{i+1} \mathbf{R}^{i} \dot{\boldsymbol{\omega}}_{i}+{ }_{i}{ }_{i} \mathbf{R}^{i} \boldsymbol{\omega}_{i} \times \dot{\theta}_{i+1}{ }^{i+1} \hat{\mathbf{Z}}_{i+1}+\ddot{\theta}_{i+1}{ }^{i+1} \hat{\mathbf{Z}}_{i+1} \\
{ }^{i+1} \dot{\mathbf{v}}_{i+1} & ={ }_{i}^{i+1} \mathbf{R}\left(\dot{\boldsymbol{\omega}}_{i} \times{ }^{i} \mathbf{P}_{i+1}+{ }^{i} \boldsymbol{\omega}_{i} \times\left({ }^{i} \boldsymbol{\omega}_{i} \times{ }^{i} \mathbf{P}_{i+1}\right)+{ }^{i} \dot{\mathbf{v}}_{i}\right) \\
{ }^{i+1} \dot{\mathbf{v}}_{C_{i+1}} & ={ }^{i+1} \dot{\boldsymbol{\omega}}_{i+1} \times{ }^{i+1} \mathbf{P}_{C_{i+1}}+{ }^{i+1} \boldsymbol{\omega}_{i+1} \times\left({ }^{i+1} \boldsymbol{\omega}_{i+1} \times{ }^{i+1} \mathbf{P}_{C_{i+1}}\right)+{ }^{i+1} \dot{\mathbf{v}}_{i+1} \\
{ }^{i+1} \mathbf{F}_{i+1} & =m_{i+1}{ }^{i+1} \dot{\mathbf{v}}_{C_{i+1}} \\
{ }^{i+1} \mathbf{N}_{i+1} & ={ }^{C_{i+1}} \mathbf{I}_{i+1}{ }_{i+1}^{i+} \dot{\boldsymbol{\omega}}_{i+1}+{ }^{i+1} \boldsymbol{\omega}_{i+1} \times{ }^{C_{i+1}} \mathbf{I}_{i+1}{ }^{i+1} \boldsymbol{\omega}
\end{aligned}
$$


Inward iterations

$$
\begin{aligned}
& { }^{i} \mathbf{f}_{i}={ }^{i+1}{ }_{i} \mathbf{R}^{i+1} \mathbf{f}_{i+1}+{ }^{i} \mathbf{F}_{i} \\
& { }^{i} \mathbf{n}_{i}={ }^{i} \mathbf{N}_{i}+{ }_{i+1}{ }^{i+1} \mathbf{R}^{i+1} \mathbf{n}_{i+1}+{ }^{i} \mathbf{P}_{C_{i}} \times \mathbf{F}_{i}+{ }^{i} \mathbf{P}_{i+1} \times{ }_{i+1}^{i} \mathbf{R}^{i+1} \mathbf{f}_{i+1} \\
& \boldsymbol{\tau}_{i}={ }^{i} \mathbf{n}_{i}^{T i} \hat{\mathbf{Z}}_{i}
\end{aligned}
$$

The dynamic expression can be written in the vector-matrix form as given below.

$$
\tau_{i}=\mathbf{M}_{i}(\boldsymbol{\Theta}) \ddot{\boldsymbol{\Theta}}+\mathbf{V}_{i}(\boldsymbol{\Theta}, \dot{\boldsymbol{\Theta}})+\mathbf{G}_{i}(\boldsymbol{\Theta})-\mathbf{J}^{\mathrm{T}} \mathbf{F}_{i}
$$

where

$\mathbf{M}_{i}(\Theta)$ is the $\mathrm{n} \times \mathrm{n}$ mass matrix of the robot leg .

$\mathbf{V}_{i}(\Theta, \dot{\Theta})$ is an $\mathrm{n} \times 1$ vector of centrifugal and Coriolis terms.

$\mathbf{G}_{i}(\Theta)$ is vector of gravity terms.

$\mathbf{J}^{\mathbf{T}}$ is is the Jacobian matrix.

$\mathbf{F}_{i}$ is the ground reaction forces of $\mathrm{i}^{\text {th }}$ foot.

\section{Reaction Force Calculation on Leg Tip}

In order to control the motion of the robot, the trunk body motion controller calculates the resultant control wrench (i.e., force and moment), that should be applied to the robot's body by its supporting legs. Therefore, one of the important issues of a legged robot's active force control is a successful distribution of its body force to the feet. For a statically stable multi-legged robot, at least three legs should be on the ground at any instant. If a three-dimensional reaction force vector is considered on each ground leg, the foot force distribution problem becomes indeterminate during the walking because of the closed chain system. Multiple solutions might exist, which can satisfy the force-moment balance criteria.

The following assumptions are used to analyze the foot forces that a walking robot exerts during walking

- The ground legs are assumed to be supporting the trunk body without any slippage on their tip points.

- The contacts of the tip of the feet with ground is modeled as hard point contacts with friction, which indicates that the interaction between the tip of the leg and ground is limited to three components of force: one normal and two tangential to the surface.

- Robot moves with tripod gait and walking with constant speed in straight line.

- The CG of the robot located at the body's geometric center.

- The robot body is level and parallel to the ground.

- The effect of inertia of swing legs on trunk body is assumed to be negligible for simplicity. Therefore, the robot's center of gravity does not change with leg movements; transfer legs exert no forces on the trunk body. 
Due to our assumptions the forces acting at the tip-point are restricted to three components, one normal and two tangential to the surface.

$$
\begin{aligned}
& \mathbf{F}_{i}=\left[\begin{array}{lll}
f_{x i} & f_{y i} & f_{z i}
\end{array}\right]^{T} \\
& \mathbf{W}=\left[\begin{array}{llllll}
F_{x} & F_{y} & F_{z} & M_{x} & M_{y} & M_{Z}
\end{array}\right]^{T}
\end{aligned}
$$

Under previous conditions, the equilibrium equations that balance forces and moments when three legs $(1,3$, and 5$)$ are in their support phase can be obtained from equation (18) as seen in Figure 9.

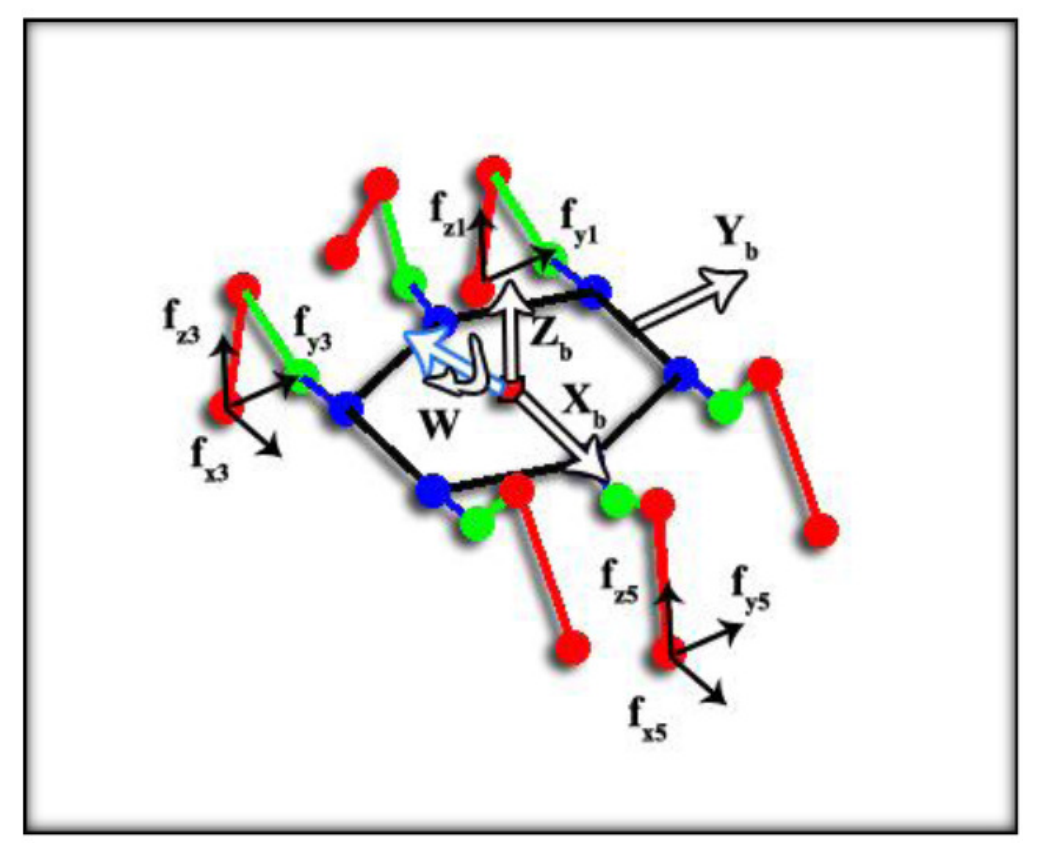

Figure 9. Schematic view showing feet contact forces acting on the robot.

$$
\begin{aligned}
& f_{x 1}+f_{x 3}+f_{x 5}+F_{x}=0 . \\
& f_{y 1}+f_{y 3}+f_{y 5}+F_{y}=0 . \\
& f_{z 1}+f_{z 3}+f_{z 5}+F_{z}=0 . \\
& -z_{1} f_{y 1}+y_{1} f_{z 1}-z_{3} f_{y 3}+y_{3} f_{z 3}-z_{5} f_{y 5}+y_{5} f_{z 5}+M_{x}=0 . \\
& z_{1} f_{x 1}-x_{1} f_{z 1}+z_{3} f_{x 3}-x_{3} f_{z 3}+z_{5} f_{x 5}-x_{5} f_{z 5}+M_{y}=0 . \\
& -y_{1} f_{x 1}+x_{1} f_{y 1}-y_{3} f_{x 3}+x_{3} f_{y 3}-y_{5} f_{x 5}+x_{5} f_{y 5}+M_{z}=0 .
\end{aligned}
$$


$\left[\begin{array}{ccccccccc}1 & 0 & 0 & 1 & 0 & 0 & 1 & 0 & 0 \\ 0 & 1 & 0 & 0 & 1 & 0 & 0 & 1 & 0 \\ 0 & 0 & 1 & 0 & 0 & 1 & 0 & 0 & 1 \\ 0 & -z_{1} & y_{1} & 0 & -z_{3} & y_{3} & 0 & -z_{5} & y_{5} \\ z_{1} & 0 & -x_{1} & z_{3} & 0 & -x_{3} & z_{5} & 0 & -x_{5} \\ -y_{1} & x_{1} & 0 & -y_{3} & x_{3} & 0 & -y_{5} & x_{5} & 0\end{array}\right]\left[\begin{array}{l}f_{x 1} \\ f_{y 1} \\ f_{z 1} \\ f_{x 3} \\ f_{y 3} \\ f_{z 3} \\ f_{x 5} \\ f_{y 5} \\ f_{z 5}\end{array}\right]=-1\left[\begin{array}{c}F_{x} \\ F_{y} \\ F_{z} \\ M_{x} \\ M_{y} \\ M_{z}\end{array}\right]$

Equation(18) can be written as follows[6]

$$
[\mathbf{C}][\mathbf{F}]=-[\mathbf{W}]
$$

The values of $F_{x}, F_{y}, F_{z}, M_{x}, M_{y}$ and $M_{z}$ are to be found for straight line motion as given below:

$\mathrm{F}_{\mathrm{x}}=M a_{x}$.

$\mathrm{F}_{\mathrm{y}}=M a_{y}$.

$\mathrm{F}_{\mathrm{z}}=M g$.

$\mathrm{M}_{\mathrm{x}}=\mathrm{M}_{\mathrm{y}}=\mathrm{M}_{\mathrm{z}}=0$.

$M=m_{\text {body }}+6 m_{\text {leg }}$ (the total mass of the robot).

The pseudo-inverse method computes the foot forces of equation (19) as:

$$
[\mathbf{F}]=[\mathbf{C}]^{T}\left[\mathbf{C} . \mathbf{C}^{T}\right]^{-1} \times-[\mathbf{W}]
$$

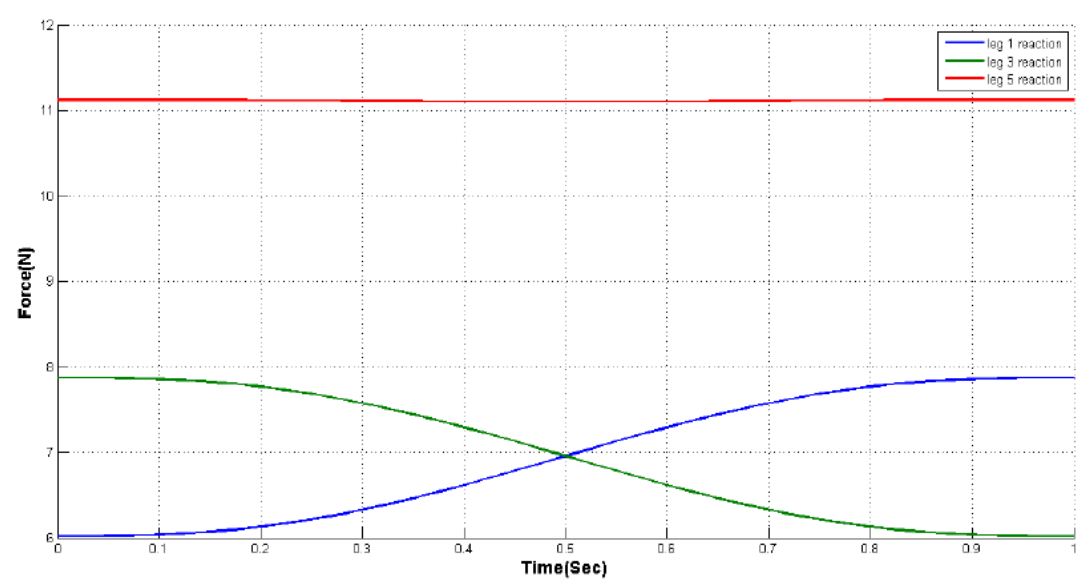

Figure 10. Simulation results of reaction forces. 


\section{RESULTS AND DISCUSSION}

In this section, simulation results for the reaction have been verified by measuring the reaction forces from the hexapod kit. In order to make hexapod robot move, control signal was sent from serial interface program (arduino serial monitor) with to arbotix via UartSBee and mini usb cable. PCB force sensor is used to measure reaction force on each leg. This sensor is connected to PC using Data acquisition system (refer to Figures 11 and 12).

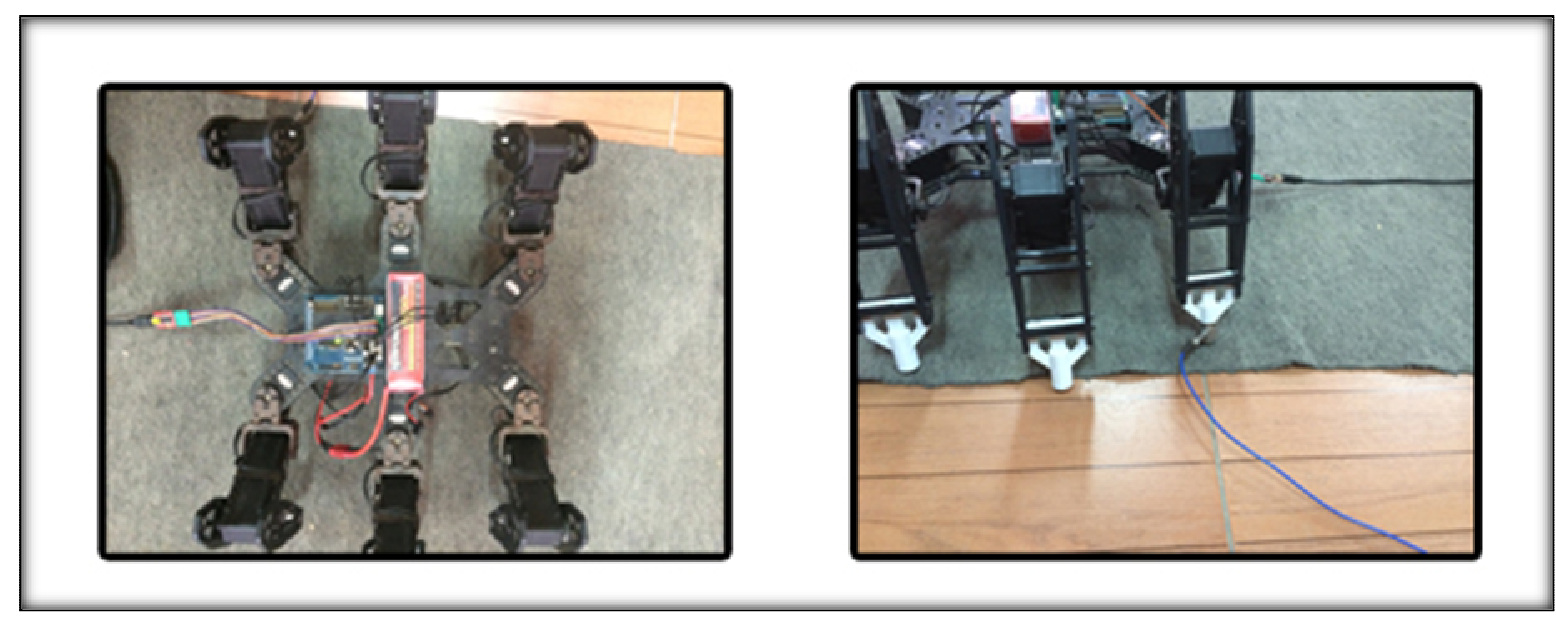

Figure 11. Reaction force measurement.

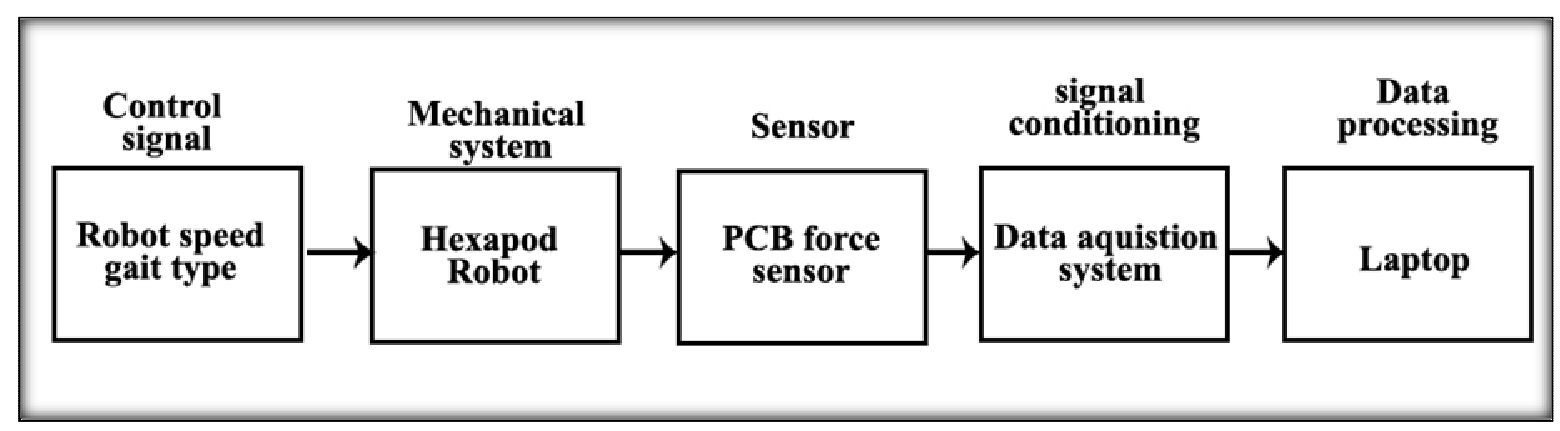

Figure 12. General scheme of measuring reaction force.

The measured reaction force on the left front leg of hexapod during one complete cycle (flying and support phases) is shown in Figure 13. The first part of the curve is labelled by number one in Figure 13 represents shock at the first of support phase then sensor dissipates, in robot tripod gait the support phase is made in two steps represented by second two peaks represented by number two. The third part shows negative reading of sensor indicating that the leg in transfer phase represented by number three.

Figure 14 illustrates measured and simulated reaction forces for the left front leg of hexapod. Difference between two curves due to the mechanical clearance of robot, 


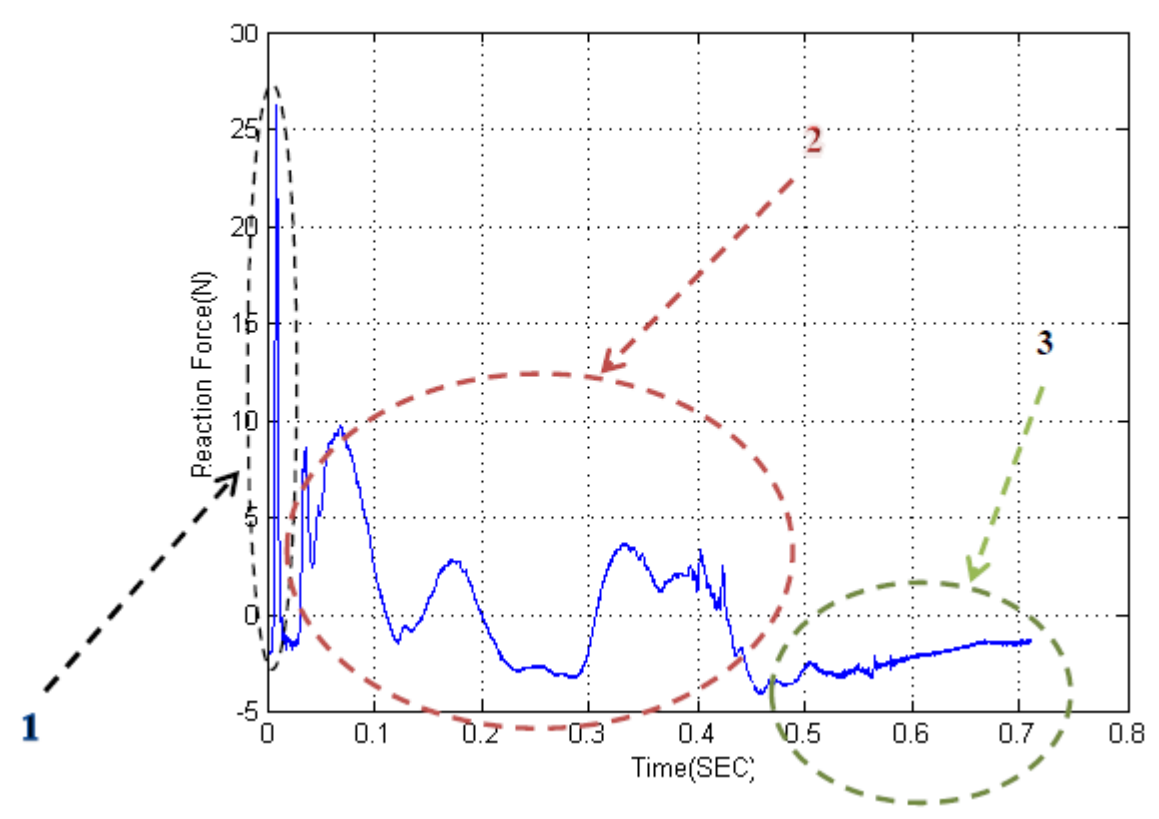

Figure 13. Leg one reaction force during one step.

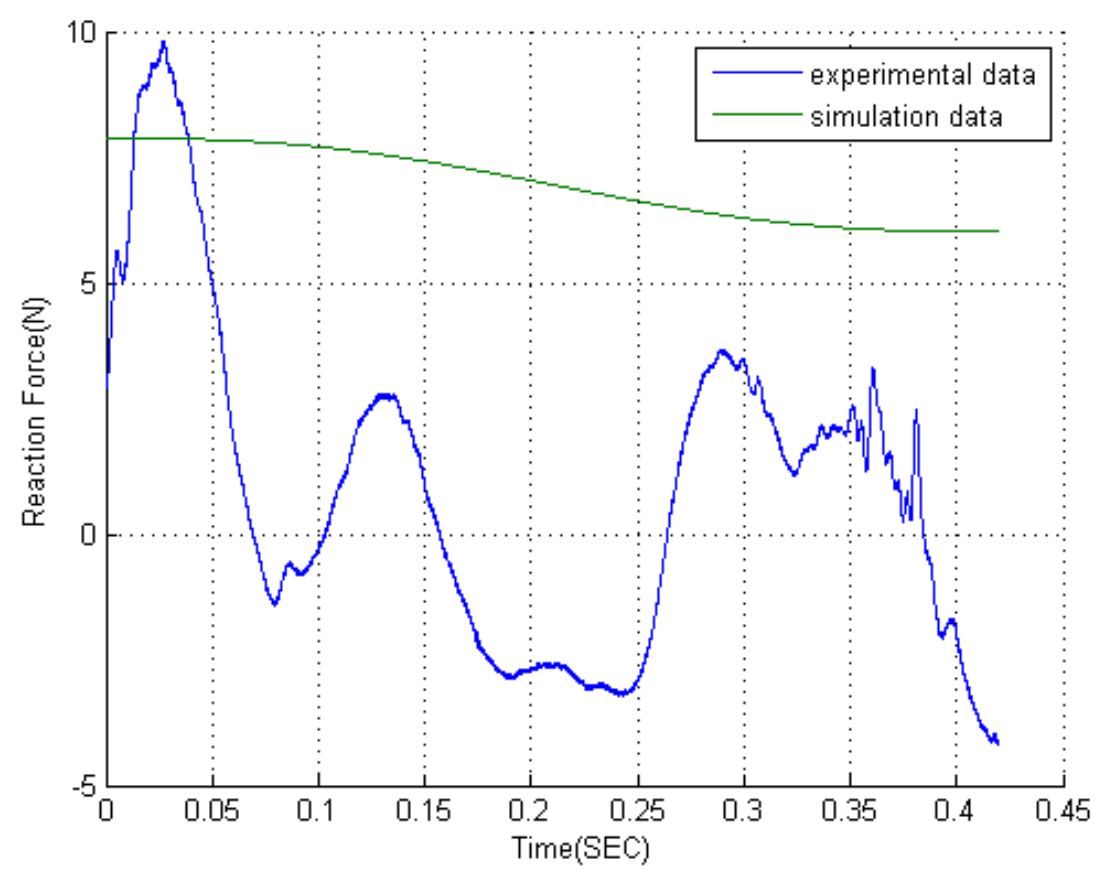

Figure 14. Comparison between measured and simulated reaction force.

slip on the ground and the physical characteristics of sensor, which can't be imported into calculation during simulation.

Walking machines are complex mechanical systems, which have been extensively studied for more than thirty years. Their principal interest is their ability to move smoothly on uneven ground and to cross obstacles that wheeled robots are not able to pass furthermore remove humans from hazardous and inaccessible environments. 
The main results of this study can be summarized as follows:

- Forward and inverse kinematic models of a six-legged robot were investigated.

- These models were verified through building GUI robot simulator using MATLAB software. This GUI facilitates the monitoring of robot CoM position, in order to sustain static stability.

- Trajectory generation was solved for both swing and support phases.

- Leg reaction forces were calculated using minimization of norm of feet forces based on least squared method and verified through experimental measurements.

\section{REFERENCES}

[1] E. Burkus and P. Odry, "Autonomous Hexapod Walker Robot," in Intelligent Systems and Informatics, 2007. SISY 2007. 5th International Symposium on, 2007, pp. 103-106.

[2] I. M. J. C. Ronde, "Simulating the "first steps" of a walking hexapod robot," Master's thesis Master's thesis, Department Mechanical Engineering Control Systems Technology Group, Technische Universiteit Eindhoven, Eindhoven, January, 2011.

[3] X. Ding, Z. Wang, A. Rovetta, and J. Zhu, "Locomotion analysis of hexapod robot," Climbing and Walking Robots, pp. 291-310, 2010.

[4] J. J. Craig, Introduction to Robotics, 2005.

[5] B. Siciliano and O. Khatib, Springer handbook of robotics: Springer, 2008.

[6] S. Agarwal, A. Mahapatra, and S. S. Roy, "Dynamics and Optimal Feet Force Distributions of a Realistic Four-legged Robot," IAES International Journal of Robotics and Automation (IJRA), vol. 1, pp. 223-234, 2012. 